\title{
NY-ESO-1-specific TCR Gene-transduced T Lymphocytes TBI-1301
}

National Cancer Institute

\section{Source}

National Cancer Institute. NY-ESO-1-specific TCR Gene-transduced T Lymphocytes TBI1301. NCI Thesaurus. Code C120557.

Human peripheral blood T-lymphocytes transduced with a retroviral vector encoding a Tcell receptor (TCR) specific for the cancer-testis antigen NY-ESO-1, with potential antineoplastic activity. Following leukapheresis, isolation of lymphocytes, expansion ex vivo, transduction, and introduction into the patient, the NY-ESO-1-specific TCR genetransduced T lymphocytes TBI-1301 bind to NY-ESO-1 on tumor cells. This may result in cytotoxic T-lymphocyte (CTL)-mediated elimination of NY-ESO-1-positive cancer cells. NY-ESO-1, a tumor-associated antigen (TAA), is found in normal testis and on the surface of various tumor cell types. 